\title{
Correspondence
}

We welcome letters to the Editor concerning articles which have recently been published. Such letters will be subject to the usual stages of selection and editing; where appropriate the authors of the original article will be offered the opportunity to reply.

Letters should normally be under 300 words in length, double-spaced throughout, signed by all authors and fully referenced. The edited version will be returned for approval before publication.

\section{How should orthopaedic surgeons respond to unsolicited e-mail?}

Sir,

I wish to congratulate Dr Wakelin and Mr Oliver on their article in the May 2001 issue entitled 'How should orthopaedic surgeons respond to unsolicited e-mail.' Mr Oliver has written and talked extensively on this topic over the last few years, providing clear guidelines for our specialty in dealing with this emerging medium of electronic communication. I agree with him but feel that we can move on from here to the next means of rapid and convenient communication: sending text messages from a mobile/handyphone. Nearly $80 \%$ of Australian surgeons carry a mobile phone and $20 \%$ access e-mail.

We have a programme in our department which sets out how to communicate with colleagues on a national and international basis. The advantages of this system are that meetings are not interrupted, the mobile phone is always available and can be held in one hand and operated with the thumb, making it easy to enter brief messages onto the screen, and the telecommunications network is at hand to organise further management of the patient.

\section{E. SHERRY}

University of Sydney

Australia.

P. H. WARNKE

University of Kiel

Germany.

1. Wakelin S, Oliver CW. How should orthopaedic surgeons respond to unsolicited e-mail? J Bone Joint Surg [Br] 2001;83-B:482-5.

\section{Authors' reply:}

Sir,

We thank the correspondents for their letter. We agree that text SMS (storage management system) messages sent to mobile phones can be used to relay medical information. Safeguards to ensure security, confidentiality and privacy need to be as rigorous as those currently used in computer standards.

We believe that wireless application protocol (WAP) mobile phones will be very useful for surgeons in the future. ${ }^{1}$ WAP is a

(C)2001 British Editorial Society of Bone and Joint Surgery $0301-620 X / 01 / 812832 \$ 2.00$

J Bone Joint Surg [Br] 2001;83-B:1208-10. standard for providing cellular phones, pagers and other hand-held devices with secure access to e-mail and text-based world-wideweb pages. WAP features the wireless mark-up language which is a streamlined version of HTML (hypertext mark-up language), used on the desktop, but adapted for small screen displays. WAP also supports hand-held input methods, such as the keypad and voice recognition. Independent of the air interface, WAP runs over all the major wireless networks in place now. It is also deviceindependent, requiring only a minimum functionality in the mobile phone unit and therefore can be used with a large number of phones and hand-held devices. It has been inhibited by narrowbandwidth connection, but forthcoming improved connection protocols will make universal access acceptable.

We believe that the future of the distribution of surgical information will be in the area of personal digital assistants (PDAs). A PDA is a hand-held computer which serves as an organiser for personal information. It generally includes at least a name and address database, a to-do list, handwriting recognition software and a note-taker. PDAs are pen-based and use a stylus to tap selections on menus and to enter printed characters. The unit may also include a small on-screen keyboard which is tapped with the pen. Data are synchronised between the PDA and a desktop computer by cable or wireless transmission. Orthopaedic education is already being delivered by PDA devices.

In the near future there will be a convergence of WAP (mobile phone) and PDA (mobile computer) devices into a small composite hand-held device. When this occurs there will be very significant advances in communications for orthopaedic and trauma surgeons.

\section{W. OLIVER, DM, FRCS (Trauma \& Orth), FRCP}

S. WAKELIN, MB ChB

Royal Infirmary of Edinburgh

Edinburgh, UK.

1. http://wapsilon.com/ (accessed 16 July 2001).

2. http://www.medscape.com/Home/Topics/orthopedics/orthopedics.html (accessed 16 July 2001).

\section{The distress and risk assessment method (DRAM)}

Sir,

I write with reference to the paper by Hobby et al entitled 'The distress and risk assessment method (DRAM)' in the January 2001 issue. $^{1}$ My concern is not with the study, its design or with its conclusion, but rather with the discussion and the list of references.

Advances in science are rarely based on one series of observations, but rather on the cumulative evidence for or against a hypothesis. If we accept the work of Main et $\mathrm{al}^{2}$ then certainly we might exclude patients for spinal surgery who have high DRAM scores. Yet, on the evidence from this paper we should not exclude patients from lumbar discectomy. Is there evidence in the literature suggesting that in other types of spinal surgery the DRAM is equally unpredictive of clinical outcome? If so, is there a case for suggesting that while psychological factors do influence outcome in spinal surgery, the DRAM method is not useful in so doing and therefore should not be applied to this group of 
patients? No comment is made as to whether any of the patients had ongoing litigation at the time of surgery. This also could have a profound effect on the DRAM score.

It seems a curious oversight that the authors failed to quote the only other reference in the literature relating to DRAM and outcome in spinal surgery. ${ }^{3}$

R. ROSS, FRCS Ed, FRACS

Hope Hospital

Salford, UK.

1. Hobby JL, Lutchman LN, Powell JB, Sharp DJ. The distress and risk assessment method (DRAM): failure to predict the outcome of lumbar discectomy. J Bone Joint Surg [Br] 2001;83-B:19-21.

2. Main CJ, Wood PL, Hollis S, Spanswick CC, Waddell G. The distress and risk assessment method: a simple patient classification to identify distress and evaluate the risk of poor outcome. Spine 1992; 17:42-52.

3. Tandon V, Campbell F, Ross ER. Posterior lumbar interbody fusion: association between disability and psychological disturbance in noncompensation patients. Spine 1999;24:17:1833-8.

\section{Author's reply:}

Sir,

We thank Mr Ross for his comments on our paper. I agree that advances in science are rarely based on one series of observations. When we planned this study we were unaware of any published articles assessing specifically the DRAM score in relation to spinal surgery, and we considered this to be important because of the growing use of this easy method of psychological assessment in spinal units.

Our study was intended to be the first of a series into various types of spinal surgery, and I only became aware of the work of Mr Ross and his colleagues, and subsequently also that of Philip Sell in Leicester, after submission of our paper. The study by Tandon et $\mathrm{al}^{1}$ is clearly of great importance, and addresses the key question concerning the use of the DRAM score in spinal surgery, namely its application to the back rather than sciatic pain. We used sciatica as a starting point because it represented a simple model with a reasonable number of cases in order to set up the method.

It is fascinating that the paper by Tandon et $\mathrm{al}^{1}$ finds that the DRAM score does not predict the outcome of spinal surgery for back pain, in view of the previous work using the various other methods of psychological assessment which have demonstrated such an influence. I agree with the question raised by Mr Ross. Does this mean that the DRAM score is of no value in assessing patients for spinal surgery? It has been my clinical impression over many years that the time-honoured teaching to avoid operation on those patients with psychological disturbance is incorrect. We all know what a profound effect the disability from severe spinal problems has in this group of patients who are at the peak of their working lives. The DRAM score reflects this whereas other methods of psychological assessment have failed, for reasons which I am unable to discern.

I also agree that a common theme in previous publications has been the adverse influence on outcome caused by ongoing litigation at the time of surgery. In our study, no such cases were knowingly included.

D. SHARP, MD, FRCS

Ipswich Hospital, UK.

1. Tandon V, Campbell F, Ross ER. Posterior lumbar interbody fusion: association between disability and psychological disturbance in noncompensation patients. Spine 1999;24:1833-8.

\section{Fractures involving splitting of the humeral head}

Sir,

I read with considerable interest the article entitled 'Fractures involving splitting of the humeral head' by Chesser et $\mathrm{al}^{1}$ in the April 2001 issue. I agree that it is essential to obtain adequate radiographs for diagnosing injuries of the proximal humerus and that good functional results can be achieved with closed reduction, or with open reduction in the presented cases.

The authors, however, have misunderstood the article by Neer ${ }^{2}$ in which he classified the head-splitting fracture as one of the fractures of the articular surface. Furthermore, when close examination is made of the Figures illustrated in the article of Chesser et al, their cases seem to be classified as posterior fracturedislocation rather than as splitting fracture. If indeed their cases are equivalent to posterior fracture-dislocations, these authors should also note the study by $\mathrm{Neer}^{3}$ in which he made the salient observation that adequate attachment and contact are generally found in patients with posterior dislocation accompanied by fracture of the anatomical neck of the humerus. In nine of ten such cases studied by us, a fracture of the humeral head which remained in such contact and was attached by a triangular metaphyseal fragment in the inferomedial part, did not undergo clinical aseptic necrosis. ${ }^{4}$ Therefore, except for patients with a completely detached humeral head, primary hemiarthroplasty does not appear to be warranted for the patient with such acute injuries regardless of age.

K. OGAWA, MD

School of Medicine, Keio University

Tokyo, Japan.

1. Chesser TJS, Langdon IJ, Ogilvie C, Sarangi PP, Clarke AM. Fractures involving splitting of the humeral head. J Bone Joint Surg [Br] 2001;83:423-6.

2. Neer CS. Displaced proximal humeral fractures. Part 1. Classification and evaluation. J Bone Joint Surg [Am] 1970;52-A:1077-89.

3. Neer CS. Prosthetic replacement of the humeral head: indications and operative techniques. Surg Clin North Am 1963;43:1581-97.

4. Ogawa K, Yoshida A, Inokuchi W. Posterior shoulder dislocation associated with fracture of the humeral anatomic neck: treatment guidelines and long-term outcome. J Trauma 1999;46:318-23.

\section{Authors' reply:}

Sir,

We thank Dr Ogawa for his interest in our paper. In 1970 Neer stated that "it is confusing to find opinions as to what constitutes a fracture dislocation" and went on to say that "a fracture dislocation occurs with a true dislocation which implies ligamentous damage and injury outside the joint". ${ }^{1}$ The confusion in terminology continues. The cases which we described involve an intraarticular split of the humeral head in which part of the articular surface may be out of the glenoid cavity and are thus by definition dislocations. However, Neer described the head-splitting fracture with the articular surface fragmented into many disconnected pieces. In seven of the eight cases which we reported there was only one fracture line and in no case was articular comminution seen.

Rare injuries are often difficult to classify, especially into a system which is known to have a significant inter- and intraobserver error $^{2,3}$ and for this reason it is important to describe the fracture, rather than to fit it into an existing system. We note with interest Dr Ogawa's report of ten cases which involved fracture of the anatomical neck. ${ }^{4}$ It is our recommendation that all fractures 
of the proximal humerus should undergo adequate radiological screening to identify the pattern. In our experience reduction, whether it be closed or open, gave a good functional result.

We agree with Dr Ogawa that primary hemiarthroplasty, which has been previously recommended, does not appear to be warranted in these patients.

T. J. S. CHESSER, FRCS (Trauma \& Orth)

P. P. SARANGI, MD, FRCS

I. J. LANGDON, FRCS (Trauma \& Orth)

Bristol Royal Infirmary

C. OGILVIE, MD, FRCS

A. M. CLARKE, FRCS (Orth)

Taunton and Somerset Hospital

Taunton, UK.

1. Neer CS. Displaced proximal humeral fractures. Part 1. Classification and evaluation. J Bone Joint Surg [Am] 1970;52-A:1077-89.

2. Sidor ML, Zuckerman JD, Lyon T, et al. The Neer classification system for proximal humeral fractures: an assessment of interobserver reliability and intraobserver reproducibility. J Bone Joint Surg [Am] 1993;75-A:1745-50.

3. Siebenrock KA, Gerber C. The reproducibility of classification of fractures of the proximal end of the humerus. J Bone Joint Surg [Am] 1993;75-A:1751-5.

4. Ogawa K, Yoshida A, Inokuchi W. Posterior shoulder dislocation associated with fracture of the anatomical neck: treatment guidelines and long term outcome. J Trauma 1999;46:318-23.

\section{Sample size and statistical power of randomised, controlled trials in orthopaedics}

Sir,

I cannot dispute the findings in the article by Freedman, Back and Bernstein, entitled 'Sample size and statistical power of randomised, controlled trials in orthopaedics' in the April 2001 issue, the conclusions of which were that most randomised trials in orthopaedics do not have adequate patient numbers to be able to detect statistically significant results, but question the authors' comments that randomised trials undertaken without a power calculation to determine the number of patients to be studied are unethical.

In the ideal situation dilemmas over treatment are best resolved by a well-conducted randomised trial. To avoid type-II errors, however, trials in excess of 1000 patients are often required, thereby necessitating a multicentre study. While this is to be encouraged, practical and financial restrictions mean that it is generally not possible. An alternative is to encourage smaller randomised trials with appropriate methods and standardised reporting of results. The results of these smaller studies can then be combined in meta-analysis, thereby achieving a study of appropriate numbers and statistical significance. Such a practice is now routine in many branches of medicine. One example of such a meta-analysis in orthopaedics was to combine the 15 random- ised trials involving 2023 cases comparing the Gamma nail with the sliding hip screw. ${ }^{2}$ Individually, no study was large enough to give valid results, but collectively clear conclusions could be made. Small trials also enable individual clinicians to have their own research projects, rather than being a small part of a large multicentre study, and such studies are easier to organise and finance. I have to disagree therefore with the conclusions of Freedman et al. Small randomised trials are not unethical, but can have a useful role in resolving dilemmas of treatment.

M. PARKER, MD FRCS Ed

Peterborough District Hospital

Peterborough, UK.

1. Freedman KB, Back $\mathbf{S}$, Bernstein J. Sample size and statistical power of randomised controlled trials in orthopaedic surgery. $J$ Bone Joint Surg [Br] 2001;83-B:397-402.

2. Parker MJ, Handoll HHG. Gamma and other cephalocondylic intramedullary nails versus extramedullary implants for extracapsular hip fractures (Cochrane Review). In: The Cochrane Library, Issue 2, 2001. Oxford: Update Software.

\section{Authors' reply}

Sir,

We thank Mr Parker for his letter in which he takes issue with our claim that it is unethical to perform randomised controlled studies with inadequate samples. We believe that it is wrong to conduct any study if it is known that it will not yield meaningful results.

Mr Parker suggests that we should "encourage small randomised trials", in the hope that they can be combined by a metaanalysis to one meaningful project. We disagree. It is much better that the investigators collaborate at the outset. Why leave it to chance that additional studies will be done and that they will be combined in a meta-analysis? There is no doubt that a metaanalysis of studies comprising 2023 cases is inferior to and makes weaker conclusions than one unified trial studying a similar number of patients. Although it is laudable that individual clinicians can "have their own research projects" it is not a sound policy to encourage them in the hope that a critical mass will be reached.

The word 'unethical' is perhaps too harsh, as the individual investigator has only the best intentions, but good intentions are often not enough. We believe that we owe our patients the best possible advice and counsel and that, in turn, demands that our studies are of appropriate power.

It may be easier to finance small studies as Mr Parker suggests, but "you get what you pay for".

\section{K. B. FREEDMAN, MD}

S. BACK, BA

J. BERNSTEIN, MD, MS

University of Pennsylvania

Philadelphia, USA 